\title{
SenseCityVity: Mobile Crowdsourcing, Urban Awareness, and Collective Action in Mexico
}

\author{
Salvador Ruiz-Correa, Darshan Santani, Beatriz Ramírez Salazar, Itzia Ruiz Correa, \\ Fátima Alba Rendón-Huerta, Carlo Olmos Carrillo, Brisa Carmina Sandoval Mexicano, \\ Ángel Humberto Arcos García, Rogelio Hasimoto Beltrán, and Daniel Gatica-Perez.
}

Keywords: mobile crowdsourcing, urban awareness, collective action, social and urban computing, Global South.

\begin{abstract}
This work describes SenseCityVity, an approach to engage and support youth of a city in Mexico to investigate, document, and reflect upon urban problems though mobile crowdsourcing. SenseCityVity focused on the development of a mobile crowdsourcing platform; the deployment of an Urban Data Challenge, co-designed by our research team and actors to collect geo-localized images, audio and video; and the analysis, appropriation, and creative use of the collected data for community reflection and artistic creation. Our approach integrates mobile technology and community practices involving a large population of young people for urban engagement. The collective action generated a new multimedia data set that is rich in terms of content and is enabling a number of studies towards the understanding of the urban landscape of cities in the Global South.
\end{abstract}

\section{Introduction}

The future of cities in the Global South depends on the ability of their populations and governments to implement frameworks for sustainable development, i.e., processes "by which people individually and collectively enhance their capacities to improve their lives according to their values and interests" [1]. Cities in Latin America face complex challenges such as violence and economic inequality. In its most recent report on the state of the world's cities, focusing on trends in Latin America and the Caribbean, the United Nations Human Settlements Program states that cities are steadily expanding, sometimes outstripping the rise in population by two to three-fold due to urban sprawl, and that $90 \%$ of the Latin American population will live in towns and cities by 2050 [2]. Countries in this world region have also booming young populations, who are not only the inheritors of significant urban problems, but also the most promising source of solutions. How can young people be incentivized to become more aware of their urban environment? How can they be supported to reach a deeper understanding of their urban problems, moving towards designing potential solutions?

Here, we describe SenseCityVity, a project developed to support young citizens in Guanajuato, Mexico to effectively define, document, and reflect on their city's problems. Our approach integrates technologies such as mobile crowdsourcing and social media, with community practices where youth are on the "driver seat" of urban

UN-Habitat www. unhabitat.org

SenseCityVity www.idiap.ch/project/sensec ityvity/ and www.cemobili.org/\#! sensecit yvity/cok8 
engagement and perception. SenseCityVity focused on three topics: (1) the development of a mobile crowdsourcing platform; (2) the deployment of a mobile crowdsourcing experiment (in the form of an Urban Data Challenge), which was codesigned by the research team and the community of young people to collect and map geo-localized images, audio and video; and (3) the analysis, appropriation, and creative use of the collected data for community reflection and artistic creation. In this paper, we share our research experience engaging with a large population of young participants in the field and the lab, and summarize the findings of a number of data analyses towards understanding the perception of youth with respect to their local urban problems.

\section{Related Work}

There is a number of related works along various thematic lines, including civic reporting systems, youth and community engagement, open mapping initiatives, and social media.

Civic Reporting Systems: One of the earliest platforms to enable crowdsourced citizen reporting and mapping of local civic issues is FixMyStreet (FMS). Reporting an issue via FMS involves providing a description of the problem, locating the problem on the map, and optionally uploading a picture to illustrate the problem. SeeClickFix is another civic reporting system. These systems engage local public authorities to ensure that the citizen reports are timely looked into and subsequently fixed. In a report analyzing 400,000 reports submitted to the FMS platform over a period of six years, it was found that only $11 \%$ of reports contain images [3]. The average response time for a report to get fixed was 66 days, and the issues reported with a photo had a tendency to get a more positive response from the local authorities, implying that the photo taking exercise itself is a strong indicator of future citizen participation. These findings support our design choice of engaging participants with geo-localized media. Our work is community oriented, which is in contrast with individual participation promoted in these existing systems.

Youth and Community Engagement: Our work is related to a number of research efforts focused on designing community-based solutions with the youth population. In [4], Poyntz describes a national research programme to explore non-profit youth media production and civic learning initiatives in Canada's three largest cities. Choi uses data to render the urban social networking of youth in Seoul [5]. Forth et al. explore design considerations for developing web portals to facilitate networking of urban residents in Mexico, South Africa, and Australia [6]. Balestrini et al. investigate the factors that are vital to develop community engagement and ICT interventions in Argentina [7]. Odendaal et al. examines the local transformative qualities of mobile telephony in Durban [8].

Open Mapping Initiatives: Our work is also related to a number of citizens mapping efforts in developing regions, including Map Kibera in Kenya and various community initiatives around the Ushahidi platform. Humara Bachpan is an organization in India
SenseCityVity's video https://www.youtube.com/w atch?v=sxUFcFCVyDA
FixMyStreet www.fixmystreet.com SeeClickFix en.seeclickfix.com
Map Kibera www.mapkibera.org Humara Bachpan http://www.humaraba chpan.org/ 
that conducts civic campaigns where groups of children create detailed "social maps" of their marginalized neighborhoods to voice their concerns about the public space in Indian slums. In the Latin American context, Google and Microsoft started an initiative to map the urban informal settlements in Rio de Janeiro, which do not currently exist on city maps. Similar efforts have been undertaken in Argentina, to improve the integration of urban spaces through mapping and monitoring Buenos Aires' marginal areas. In a study conducted in Brazil, Offenhuber et al. mapped the spatial organization of waste collection though mobile technology [9].

Mobile data in everyday life. Our work also has connections with studies on how people go about their daily routines and interact with the world through mobile devices. Korn and Voida discuss a theoretical framework that advocates for the design of civic engagement infrastructure supported by $\mathrm{HCl}$ tools that provoke contestation through embedded use in the everyday life of citizens [10]. Le Dantel et al. study how data from a smartphone app can be incorporated into urban planning events [11]. Kitner and de Wet describe an experience in Johannesburg, aimed at tracking and studying the everyday behaviors and practices of urban traders and a group of university students equipped with smartphones [12].

Social media. Finally, social media is increasingly used as a medium to voice urban concerns. In the specific Mexican context, Twitter has been actively used as a channel to disseminate eyewitness reports of drug-related crimes in several cities [13].

It is clear that an integrative approach, where multiple sources of information and a variety of analysis methods are combined, would allow painting a more accurate picture of how youth perceive their own urban environment. Our work integrates community practices, mobile crowdsourcing for data collection, and mixed methods for analysis (both quantitative data analysis and interviews) to advance the understanding of how working class youth populations in Mexico can be supported to document, characterize, and reflect on urban concerns, while generating previously unavailable data resources, and contributing to the creation of suitable conditions to transform the collected data into insights about urban issues, and to debate community-oriented solutions.

Beyond the creation of maps, our approach encourages the use of the collected data for artistic and educational endeavors by the youth communities. This approach helped youth reconnect to the tangible reality of their city. In our opinion, the contribution of our work is the demonstration that the integration of existing methods and best practices in ubiquitous computing and $\mathrm{HCl}$ and the understanding of a concrete social context can result in a participatory framework that allows phone-based sensing for surveying and articulating urban challenges, and enables participants to share their experiences and reflections in a variety of ways. 


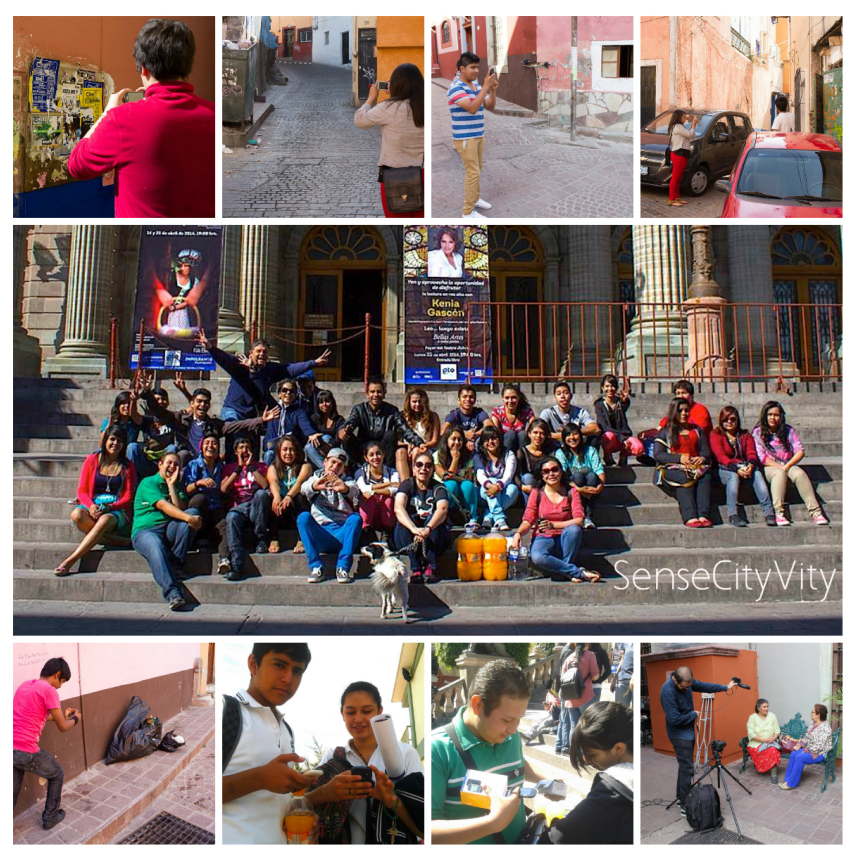

Figure 1. Urban Data Challenge (UDC) implemented by students and the research team.

\section{Guanajuato City as an Urban Lab}

Guanajuato City (pop. 170,000) is the capital city of Guanajuato state in Central Mexico. Guanajuato grew as one of the most important mining towns in the XVI Century, which developed into a city of great historic significance. Over the years, a rough geographical terrain led to an urban landscape that has many narrow and winding alleys and streets, originally constructed on the side of streams running downhill. Various streets, where rivers once flowed, run underground, and long tunnels carved in solid rock connect various zones of the city. The traditional architecture and unique construction style characterizing the urban space, the large number of historic monuments and museums, and two of the most important international cultural festivals that occur yearly, make Guanajuato City attractive for tourism. The city is surrounded by small towns and communities, and is also an important connection to other cities in Guanajuato State. However, like numerous cities in the Global South, historical patterns of disordered urban growth, characterized by the fast expansion of the borders of the city and the establishment of informal settlements, have resulted in a complex urban environment where large socioeconomic disparities pervade.

\section{Methods}

As part of the SenseCityVity project, we use a methodology to explore Guanajuato's intricate urban landscape through five main activities that enabled the integration of youth work supported by mobile and social technologies: (1) development and deployment of a mobile crowdsourcing platform; (2) recruitment of a population of student volunteers; (3) co-design of a mobile crowdsourcing experiment -- an Urban 
Data Challenge (UDC); (4) implementation of the UDC; and (5) creative use of collected data by participating volunteers.

Mobile Crowdsourcing Application. For gathering data during the project, we designed and developed an Android-based mobile application. The application recorded the location traces of students in the background and facilitated them to take pictures and videos, while mapping Guanajuato. All the generated data was automatically and synchronized with our backend server. The mobile application was active only during the UDC to prevent collection of participant's personal data. Data collection used best practices to satisfy the requirements of anonymized data management [16].

Recruitment of Volunteers. A population of volunteers (students between 16-18 years of age) was recruited from the Centro de Estudios Científicos y Tecnológicos de Guanajuato (CECYTE-G), a high school that provides education in science, technology and humanities to working class youth in Guanajuato City and surrounding suburbs. The recruitment process took four weeks of intensive work of our research team with school authorities, teachers, parents and students. Our project goals were presented to the community during a series of weekly workshops that emphasized the importance of the collective participation of citizens to understand and address pressing urban issues, and how social and mobile technologies could be used to empower people to achieve such goals. Our population of volunteers consisted of 10 teams of 10 members each. Teams were encouraged to actively seek support from their classmates for achieving their goals, so that a larger community could be involved in the project. At the end of the fourth week, participating teams and their work plans were presented during a plenary session to 600 students, teachers and team leaders' parents. At the end of the session, the community was motivated and eager to support our research effort to better understand the urban environment in which they live, the urban problems they face on a daily basis, as well as to reflect on how to develop communitybased solutions to address such problems. To incentivize student participation, registered volunteers were allowed to accumulate the time spent in SenseCityVity's activities towards their social service requirement.

Experiment Co-design. Our research team and the participating volunteers designed the mobile crowdsourcing experiment. The co-design was conducted during a series of four weekly workshops and study groups that led to discussions about mobile and social media technologies for the common good, ethics, data privacy, personal safety, urbanism, and basic techniques in film-making and photography. The workshops intended to spark the curiosity of the volunteers to address the challenges of our mobile crowdsourcing experience, keeping in mind that youth often use and transform technology in the most innovative ways. During the last workshop, participating teams developed a chronogram of the activities that would be conducted during the UDC. Teams were instructed to focus on urban challenges negatively affecting the city landscape instead of focusing on both positive and negative aspects. Therefore, each team defined one or two urban problems they wanted to document, and the specific approach to conduct their investigation. The urban problems highlighted during the 
co-design workshops ranged from garbage in the streets and alleys, crime and vandalism, worn public infrastructure, graffiti, prevalence of alcoholism and drugs, to mention a few. The project also integrated the use of social media (Facebook and Twitter) to maintain communication with the student community, while collecting additional images through a dedicated channel. Throughout the rest of the paper, an "urban concern" points to one of the urban problems discussed during the co-design workshops.

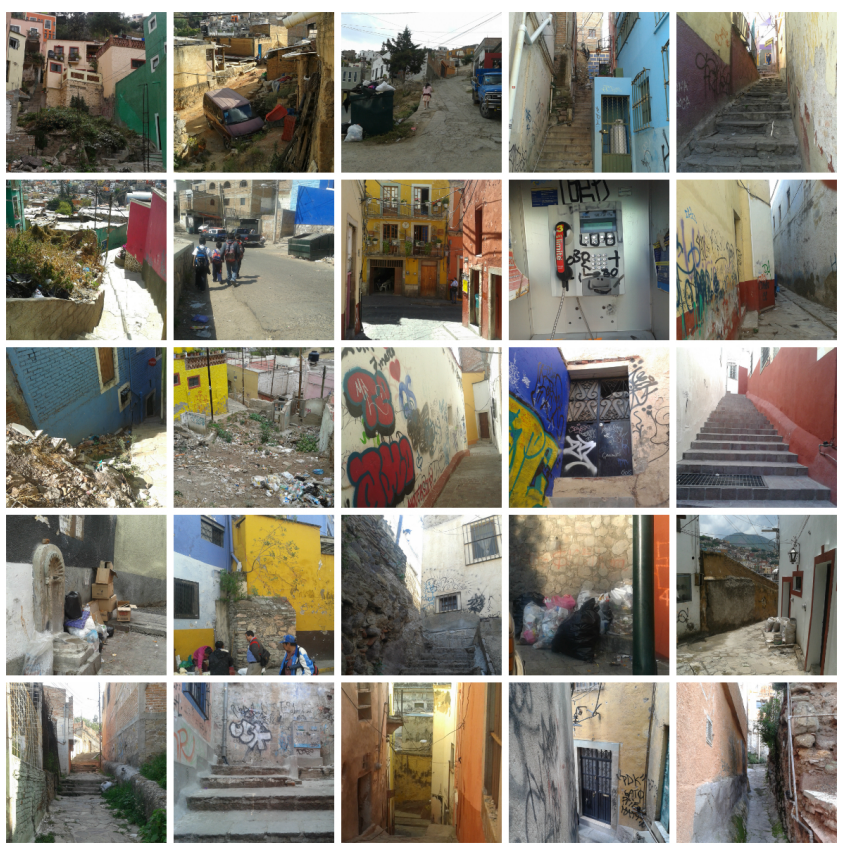

Figure 2. Sample photos of Guanajuato City taken during the UDC.

Urban Data Challenge. The UDC (Figure 1) lasted for eight weeks and consisted of two main activities - data collection in the field by student volunteers; and an online crowdsourcing experiment where impressions about areas in Guanajuato City were collected using online questionnaires. Participating teams were encouraged to conduct data collection during the week (with the condition of a signed parental consent form); however, fieldwork was mainly conducted during weekends. On these weekend gatherings, members of our research team led a review session to reinforce the key ideas discussed during the workshops and to check each team's work plans for data collection. This check was necessary to ensure that student teams did not visit unsafe parts of the city.

After the review session was finished, each team moved independently to document the various parts of the city using mobile phones. Android-based smartphones were given to each team; however, students also used their own phones for data collection. Photo taking was considered a necessary practice to map Guanajuato city, given that other available imagery mediums (like Google Street View, GSV) is often incomplete or erroneous. In a preliminary study, we demonstrated that in some areas of Guanajuato, 
as much as half of the data from GSV have limitations [14]. An animated map of the exploration routes followed by students (based on geo-localized images) during the UDC is shown in the link listed on the sidebar. During the UDC, we also conducted a crowdsourcing experiment where 177 student volunteers (who did not participate in the fieldwork) provided their impressions of different areas in Guanajuato along various physical and psychological dimensions using images as stimuli. Details of this research have been described in [14].

Film Festival. With the slogan "Observe, feel, and share!" the SenseCityVity film festival provided the student community with the opportunity to make and show short documentaries (4-5 minutes duration) that they created using the data collected during the UDC. The festival took place in February 2015. Documentary topics included poverty and safety, artistic graffiti, urban and social issues, graffiti in city alleys, and garbage on the streets of Guanajuato. The film festival had three goals. The first one (at time of recruitment) was to funnel the festival as an incentive for students to participate in the project by giving them a motivation to use their own data for a common purpose. The second and third goals (during the festival itself) were to open a discussion within the broad community about the use of mobile technology to document pressing urban problems, and to reflect as a group about possible community-based solutions to some of these problems.

\section{Results}

The collected data is rich in terms of content, and is enabling a number of qualitative and quantitative studies, which are summarized below.

Collected data. A total of 277 student volunteers registered to the UDC (100 of which participated in the fieldwork, and 177 in the online crowdsourcing experiment). About 100 non-registered volunteers (relatives, classmates, friends, and teachers) additionally participated during the UDC activities. Volunteer's work during the UDC produced over 7,000 geo-localized images and 380 videos captured from the perspective of a pedestrian who is exploring the city (Figure 2). Data also includes 20 audio recordings of informal interviews, 5,300 GPS locations, 9,027 impressions of urban spaces (collected during the online crowdsourcing experiment), 14 short video documentaries, and 100 Facebook posts. During the UDC, around 19,000 generic geo-tagged tweets were also collected between 2014 using the Twitter API.

Video-recorded Interviews. Of the 380 videos collected during UDC, 200 contain informal interviews with pedestrians on the streets of Guanajuato. Of the 200 interviews, 180 followed a structured interview format, where the interviewees (108 males, 72 females) were asked three questions. (1) What are the urban problems that Guanajuato inhabitants face on a daily basis? (2) What are the urban problems that affect you the most? (3) What are possible solutions to these problems? Structured interviews have an average duration of 6 minutes each. The remaining 20 interviews
Map of exploration routes

https://centerformobilelife.c artodb.com/viz/143c94f42b32-11e5-80bd0e0c41326911/public_map

Film festival http://www.idiap.ch/p roject/sensecityvity/fil m-festival 
were audio-based since the interviewees did not want to be filmed. The 20 audio recordings also contained conversations addressing urban issues.

Video of Urban Scenes. Additionally, UDC participants recorded 180 videos to illustrate and capture urban issues (such as a large wall with painted graffiti on a bridge or a tunnel) or show relevant events (such as a bumpy ride in a city bus or a pedestrian crossing a dangerous street). Collected videos last between 20 seconds up to 10 minutes. About one third of these videos include comments from the person who is recording it, which makes these videos valuable to understand how students feel about their city. This kind of fieldwork, which requires direct interaction with people on the street, offered a unique opportunity for students to: (1) have a first-hand account of the dynamics of urban landscapes of Guanajuato, and (2) reconnect to the tangible reality of their city through the lens of their mobile device.

Mapping urban concerns. From a quantitative point of view, one of the first tasks was to create heat maps to identify areas where participants documented their respective concerns about specific urban issues. We created these maps by applying density estimation techniques to GPS locations embedded in the geo-tagged images collected during the UDC. The main areas of concern were detected in traditional neighborhoods, plazas, historic alleys, and central avenues, as shown in red in Figure 3a. One could also wonder how the UDC data compares to data from mobile social media like Twitter, which is increasingly being used as a source to analyze cities [15]. The information provided by the UDC heat map describes patterns that differ from those obtained by aggregating geo-tagged tweets for the same geographical area (Figure 3b). Specifically, Twitter activity is low in several areas of Guanajuato where the UDC teams detected urban concerns, and as expected, high in the touristic areas of the city and close to the main campus of the University of Guanajuato. Areas of urban concern might be digitally invisible in social media channels like Twitter, showing the relevance of our approach as a complementary tool to map urban spaces in developing cities. 


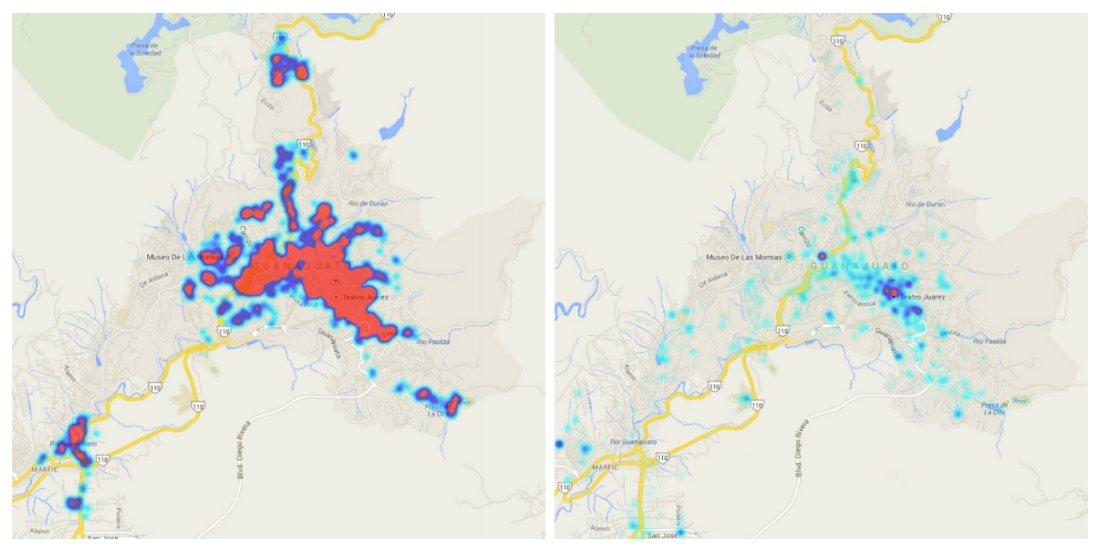

(a)

(b)

Figure 3 (a) Heat map computed using geo-localized UDC images showing the areas marked as being of concern (in red). (b) Heat map computed using geo-localized tweets by general Twitter users in Guanajuato. Red color indicates high density, while blue denotes low density.

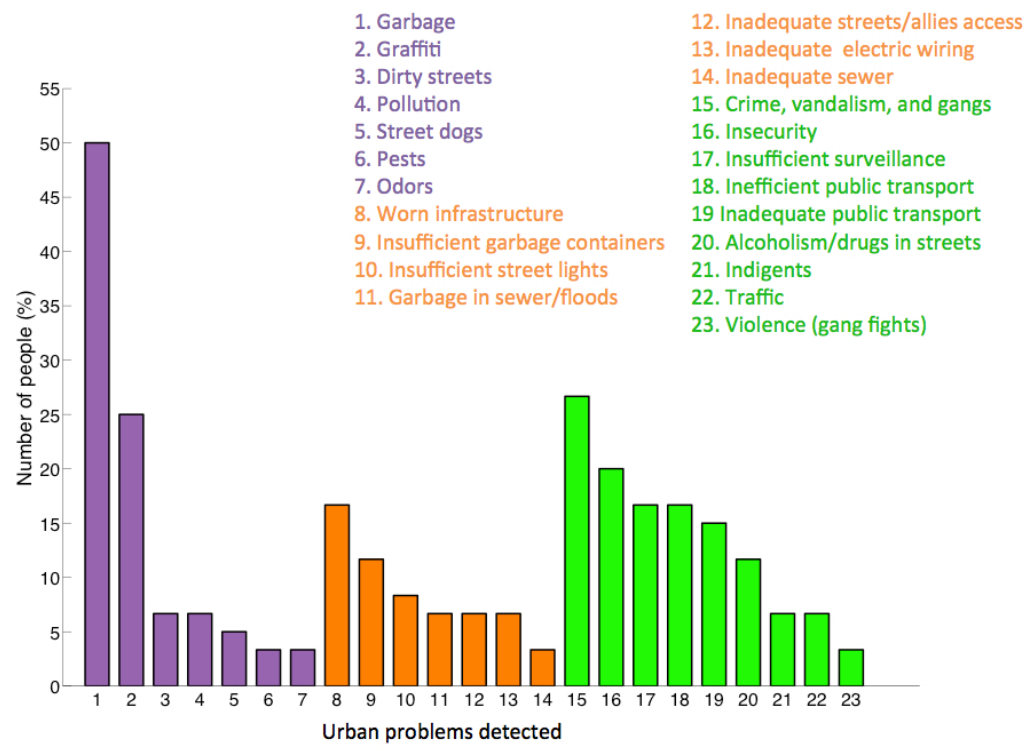

Figure 4. Urban problems detected by UDC participants, which are further divided in three categories: the image of the city (purple), infrastructure issues (orange), and the quality of city life (green).

Emerging themes. To study the state of Guanajuato City and explore the various themes captured by UDC participants, we conducted a qualitative study by manually coding the set of interviews and urban videos into three main categories: 1) videos describing the image of the city showing littered garbage, graffiti, street dogs, etc.; 2) 
videos showing infrastructural problems ranging from insufficient street lights and garbage containers to inadequate street access; and 3) videos highlighting the quality of city life from vandalism, alcoholism and drugs to insufficient public transportation. In Figure 4, we plot the number of participants who described a specific problem as per these three categories.

From Figure 4, it is clear that trash accumulation and mismanagement is identified as a serious concern that produces odors and pests, severely deteriorates the city appearance, and blocks drainage and sewer pipes especially during the rainy season. Although some inhabitants think that "the people in charge of collecting the garbage in the city are not able to do it on time, and the garbage containers often overflow polluting the environment," others believe that "... the garbage problem in the city is a people's problem. We are responsible as a community to address it". Besides the garbage problem, people are also concerned about the worn infrastructure pervasive in the city. It is not uncommon to find high-powered electric wires installed too close to rooftops leading to serious hazards.

Participants also perceived an increased sense of insecurity in many city neighborhoods and suggested increased police surveillance of streets and alleys. One participant noted: "The problem here is that there is a lot of insecurity, in the alleys, outside the downtown area. At night, there are people drinking and smoking marihuana in the street alleys. We used to play by the Hidalgo market every day. But now we are limited because of insecurity; things can be complicated and we are limited to go outside."

Over the last 20 years, the population growth in Guanajuato City and towns nearby has resulted in complex urban mobility patterns and insufficient and inadequate transportation. This issue affects the daily routines of participants, as one of them commented: "Transport does not come in time or is very scarce, or things like that, which affect us to get to school; this problem affects me directly and think affect the majority of us". Visual inspection of the geo-localized images suggests similar themes to those obtained via coding interviews and videos (Figure 4), though detailed image coding and further analysis will be carried as part of future work.

It is worth mentioning that the SenseCityVity team working in the field observed problems that were not reported by the UDC participants. For instance, the downtown area consists mainly of long winding alleys running hundreds of meters up hill. Since city lacks infrastructure for the disabled and elders, a significant number of them spend a fair amount of time walking the alleys for daily chores. However, UDC participants found that most of the interviewed elders did not complain about accessibility problems. Rather, they expressed their dissatisfaction on vandalism, safety or garbage in streets. Our team also found increased noise levels and air pollution in Guanajuato's street tunnels, which have a considerable amount of pedestrian traffic, in addition to vehicular traffic. 
Overall, our qualitative study suggests that participants are aware of both the beauty of their city and the key role that citizens should play in addressing urban issues; however, there is a general concern regarding the urban issues that are causing a gradual deterioration of the urban landscape, as well as of the need of more citizen and governmental involvement to revert this trend.

Potential Implications. A general consensus among participants with regard to possible solutions to the urban issues is that citizens and government need to work together. An historic trend in Mexico, in which citizens avoid their civic responsibilities and government institutions are oblivious to the needs of people, needs to be replaced by one in which government and citizen initiatives are put together through effective communication channels. Several of the observed problems can be directly addressed by citizen engagement, and do not require a significant amount of external funding. However, it was clear that many others require direct government intervention. More than half of the people who were interviewed during the UDC still believe that government should be responsible for social transformation. For this reason, one of the main conclusions of SenseCityVity is that it is important for citizens to create effective ways to inform, communicate, and discuss urban problems with the other inhabitants, in order to emphasize that it is through citizen awareness and engagement that people can collectively enhance their capacities to improve their lives.

\section{Conclusion}

The complex patterns of urban growth in developing cities hinder the ability of their inhabitants to achieve suitable conditions for holistic development. In this regard, SenseCityVity has demonstrated that in a specific local context in Mexico, it is possible to co-develop and apply technologies based on mobile crowdsourcing to support youth so they can effectively define, document, and reflect upon their own urban problems. This was achieved with the combination of citizen-contributed data and quantitative and qualitative approaches, which reveal what existing problems are perceived as more prevalent or go unnoticed, how these problems are geographically stratified, and what are the population's general impressions of their own urban space. However, a valid and relevant question regarding our proposed approach relates to the implications on deploying similar campaigns (with limited and incomplete data) to understand Latin American cities, and ultimately how this kind of work can be used to support city inhabitants to propose effective solutions.

We suggest that, in order to be useful for studying cities in Latin America, future approaches must be based on a methodology that meets at least five requirements: (1) be easily deployable in the field; (2) be able to facilitate data collection to complement traditional data sources (3) be capable of engaging local population from different segments and demographics of society; (4) be applicable to a variety of urban landscapes (i.e., cities with distinct features); and (5) be sustainable. 
After the completion of the first phase of the project, our research has moved into two different directions. First, we have taken our approach to multiple cities in the state of Guanajuato. Second, we have engaged people from diverse segments of the society, including young adults and elderly people. In addition, our methodology was recently adopted by other academics in different cities of Mexico, including Leon (in Guanajuato State) and Merida (in southeast Mexico.) These new partnerships will allow us to test our work in other conditions.

Acknowledgments. SenseCityVity was supported by the EPFL Center for Cooperation and Development (CODEV), the SNSF Youth@Night project in Switzerland, by the Instituto Potosino de Investigación Científica y Tecnológica (IPICYT) and CONACYT's project 247802 in Mexico. We acknowledge the work of Héctor Gómez Vargas, Victor Islas Adrán García, Rodolfo Hurtado, and Enrique Castro for producing the SenseCityVity-Leon video. We also acknowledge the enthusiastic participation of CECYTE students, teachers and and school authorities.

\section{References}

[1] M. Castells and P. Himanen. "Re-conceptualizing development in the global information age." Oxford University Press, 2014.

[2] State of the World's cities 2011-2012: Bridging the Urban Divide. United Nations Human Settlements Programme.

[3] M. Sjoberg, J. Mellon, and T. Peixoto, "The effect of government responsiveness on future political participation," World Bank Group, Digital Engagement Evaluation Team, Feb. 2015.

[4] S. R. Poyntz, "Public Space and Media Education in the City." Current Perspectives in Media Education: Beyond the Manifesto, 2013.

[5] J. H Choi. "The city, self and connections." Youth, Society and Mobile Media in Asia, 2010.

[6] M. Foth, V. M. Gonzalez, and W. Taylor. "Designing for place-based social interaction of urban residents in México, South Africa and Australia." In Proc. $\mathrm{OzCHI}$, 2006.

[7] M. Balestrini, J. Bird, P. Marshall, A. Zaro, and Y. Rogers, "Understanding sustained community engagement: a case study in heritage preservation in rural Argentina." In Proc. ACM CHI, 2014.

[8] N. Odendaal. "Space matters: the relational power of mobile technologies." Urbe, Rev. Bras. Gest. Urbana, 2014. 
[9] D. Offenhuber and D. Lee. "Putting the informal on the map: tools for participatory waste management." In Proc. PDC, 2012.

[10] M. Korn and A. Voida, "Creating friction: infrastructing civic engagement in everyday life." Aarhus Series on Human Centered Computing, Vol. 1, No. 1, 2015.

[11] C. A. Le Dantec, M. Asad, A. Misra, and K. E. Watkins, "Planning with Crowdsourced Data: Rhetoric and Representation in Transportation." In Proc. ACM CSCW, 2015.

[12] K. R. Kitner and T. de Wet, "Big data, big city." ACM Interactions Magazine, Vol. 22, No. 4, pp. 70-73, Jul. - Aug. 2015.

[13] A. Monroy-Hernández, M. De Choudhury, E. Kiciman, D. Boyd, and S. Counts. "The New War Correspondents: The Rise of Civic Media Curation in Urban Warfare." In Proc. ACM CSCW, 2013.

[14] S. Ruiz-Correa, D. Santani, and D. Gatica-Perez. "The Young and the City: Crowdsourcing Urban Awareness in a Developing Country." In Proc. Urb-loT, 2014.

[15] R. Lee, S. Wakamiya, and K. Sumiya. "Urban area characterization based on crowd behavioral lifelogs over Twitter." Personal and ubiquitous computing. Vol. 17, No. 4, pp 605620, Apr. 2013

[16] J. K. Laurilla, D. Gatica-Perez, I. Aad, J. Blom, O. Bonet, T. M. Tri Do, O. Dousse, J. Eberle and M. Miettien. "From big smaarthphone data to worldwide research: The mobile data challenge. Pervasive and Mobile Computing, Vol 9, Issues 6, 2013, pp. 752-771. 


\section{Author list (short bios \& emails)}

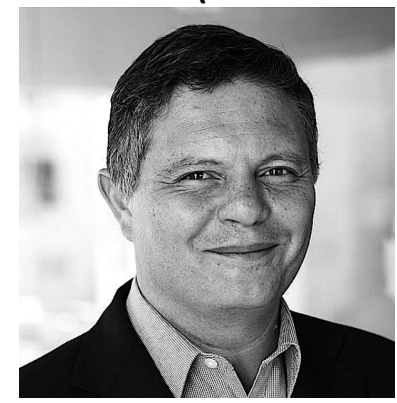

1. Salvador Ruiz Correa is Adjoint Professor and Researcher at the Instituto Potosino de nvestigación Científica y Tecnológica (IPICYT) and codirects the Center for Mobile Life (Ce Mobili) research initiative in México. His research interests include machine learning and computer vision applications to social computing in development contexts, data for social good, and citizen innovation. He is member of the SNI-CONACYT and IEEE.

\section{E-mail: src@cmls.pw}

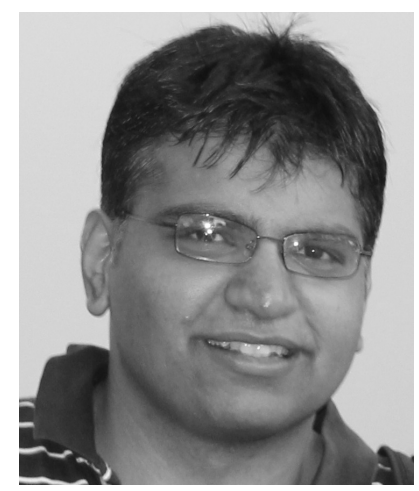

2. Darshan Santani, is a PhD student at École Polytechnique Fédérale de Lausanne (EPFL) and the Social Computing Group at Idiap Research Institute in Switzerland. His research interests include ubiquitous computing, social computing and social media.

\section{Email: darshan.santani@idiap.ch}

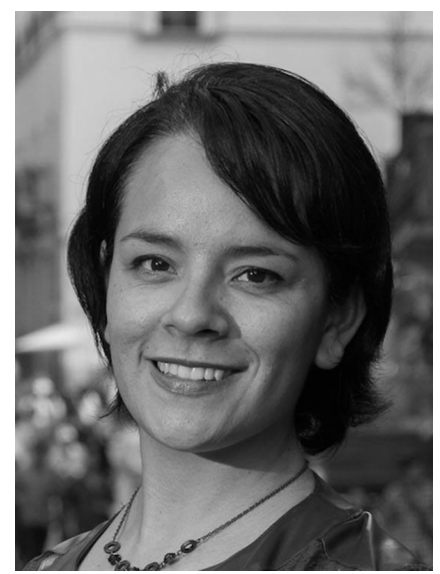

3. Beatriz Ramírez Salazar has a Master's degree in arts and is member of CeMobili.Arts group.

\section{Email: beramisa@yahoo.com}




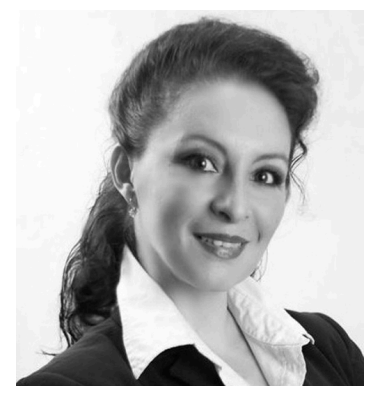

4. Itzia Ruiz Correa is a MA student at the University of Guanajuato (UG) and member of Ce Mobili.Media group.

Email: itziaruizcorrea@gmail.com

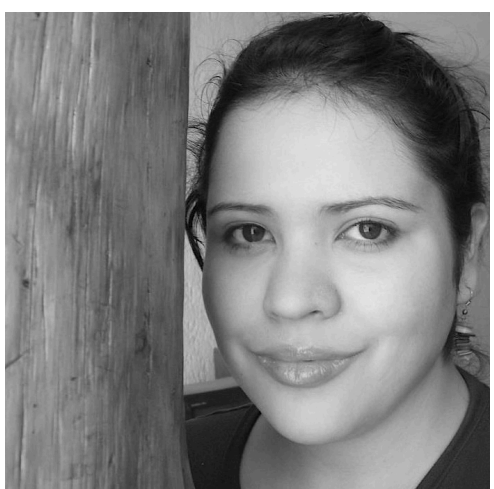

5. Fátima Alba Rendón-Huerta is a PhD student at the University of Guanajuato (UG) and member of Ce Mobili.Thinkers group.

Email: albafatima@mail.com

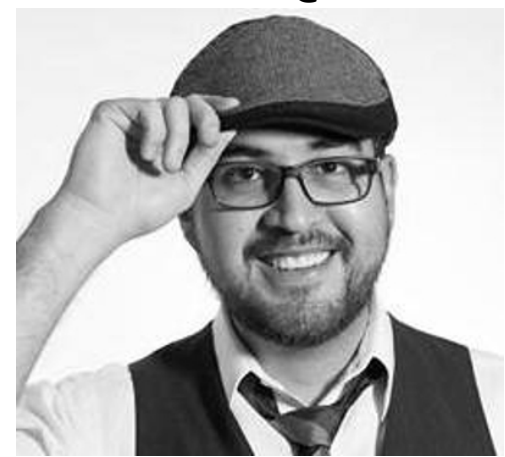

6. Carlo Olmos Carrillo has a Master's degree in Visual Composition, member of CeMobili.Arts group and founder of Producciones Huapango.

Email: locarco@gmail.com 


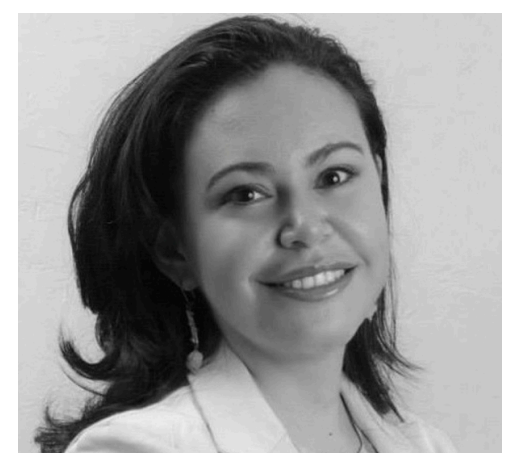

7. Brisa Carmina Sandoval Mexicano, is a Psychologist at Contacto con Empatía in Guanajuato City, and member of Ce Mobili.People group.

Email: brisacarmina@gmail.com

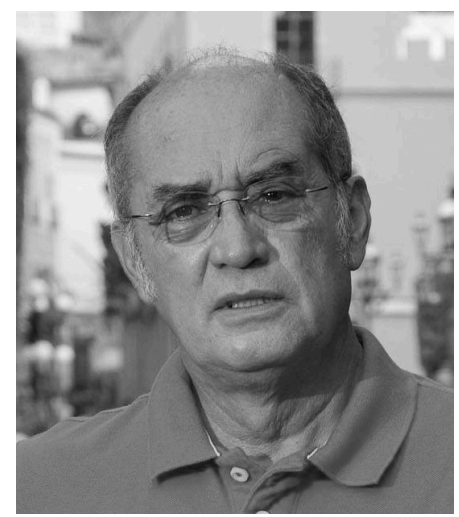

8. Ángel Humberto Arcos García is an Architect and PhD in Visual Arts. He is member of ICOMOS International, Mexico.

\section{Email: paisajeynaturaleza@gmail.com}

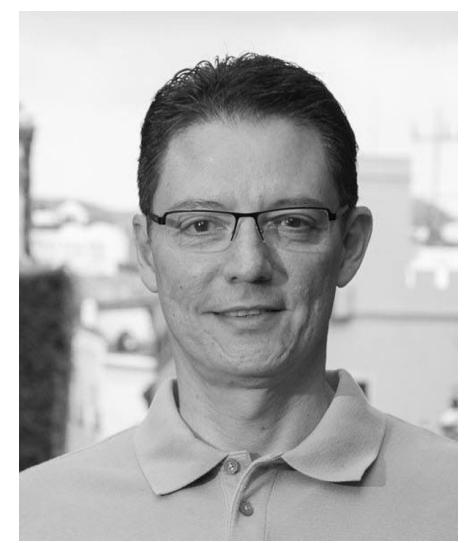

9. Rogelio Hasimoto Beltrán received his Ph.D. degree from the University of Delaware, USA, in 2001. After his Ph.D, he spent two years at Akamai Tech. as a Senior Software Engineer. Since 2003 he has served as an associate researcher in the Department of Computer Science at the Center for Research in Mathematics (CIMAT), México. His research interests include image/video processing and compression, robust multimedia transmission and multimedia encryption. He is member of the IEEE association. 


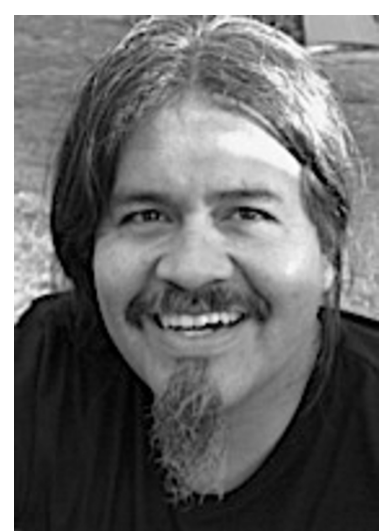

10. Daniel Gatica-Perez, Idiap-EPFL, Switzerland. directs the Social Computing Group at Idiap Research Institute and is Professeur Titulaire at the École Polytechnique Fédérale de Lausanne (EPFL) in Switzerland. His research interests include social computing, ubiquitous computing, and social media. He is a member of the IEEE.

\section{E-mail: gatica@idiap.ch}

